\title{
EMOCIONES Y SIGNIFICADOS EN LA AUTOBIOGRAFÍA: REFLEXIONES A PARTIR DE UN CASO WICHÍ (GRAN CHACO)*
}

\author{
MEANING AND FEELING IN AUTOBIOGRAPHY: A WICHÍ CASE (GRAN CHACO)
}

\author{
Zelda Alice Franceschi ${ }^{1}$ \\ Università degli Studi di Bologna
}

Recibido: 7 de diciembre de 2018; Aceptado: 11 de febrero de 2019; Publicado online: 28 mayo de 2019

\begin{abstract}
Cómo citar este artículo / Citation: Franceschi, Zelda Alice: «Emociones y significados en la autobiografía: Reflexiones a partir de un caso wichí (Gran Chaco)». Disparidades 74(1): e002e. doi: <https://doi.org/10.3989/dra.2019.01.002.05>.
\end{abstract}

RESUMEN: Se proponen algunas reflexiones metodológicas sobre la autobiografía indígena y la antropología de las emociones a través de la presentación de un caso etnográfico. El relato autobiográfico crea un vínculo, a menudo muy fuerte, entre el antropólogo y su testigo, que lleva a ambos a prestar especial atención a los dispositivos emocionales que, de repente, se convierten en un instrumento de comprensión del aparato cultural. En este caso, se sugiere una lectura particular de algunas reglas culturales relacionadas con el parentesco y la vida social.

PALABRAS CLAVE: Autobiografías; Parentesco; Antropología de las emociones; Chaco; Wichí.

ABSTRACT: The aim of this essay is to propose some methodological reflections on indigenous autobiography and on the anthropology of emotions through an ethnographical case study. The autobiography creates a link that can often be very strong between the anthropologist and the informant. This can lead both to pay particular attention to emotional aspects, which therefore become tools for understanding the cultural system. Out of this case study what has emerged is a particular reading of certain cultural practices related to kinship and life in society.

KEYWORDS: Autobiographies; Kinship; Anthropology of Emotions; Chaco; Wichí.

Copyright: ( 2019 CSIC. Este es un artículo de acceso abierto distribuido bajo los términos de la licencia de uso y distribución Creative Commons Reconocimiento 4.0 Internacional (CC BY 4.0).

\footnotetext{
* Un agradecimiento especial a Rodrigo Montani por su generosidad, sus comentarios y sugerencias. A Carmen Gregorio Gil por sus estímulos intelectuales que siempre me ofrece.

1 Correo electrónico: zelda.franceschi@unibo.it. ORCID iD: <https://orcid.org/0000-0001-6090-4755>.
} 


\section{BREVES APUNTES METODOLÓGICOS}

Autobiografía es un término que no debe engañarnos. David Brumble (1988 y 1985) y Arnold Krupat (1985), los historiadores más reconocidos de las autobiografías indígenas norteamericanas, han señalado por qué la autobiografía en el contexto indígena representa un oxímoron. Si de hecho el panorama norteamericano nos ha ofrecido un amplio repertorio de relatos autobiográficos ${ }^{2}$ y una lectura crítica de los peligros que conlleva el uso del dispositivo autobiográfico $^{3}$, es solo recientemente que en el contexto de las tierras bajas de Sudamérica surgieron autobiografías, historias de vida y biografías indígenas ${ }^{4}$.

Los problemas clásicos que habían obsesionado a los antropólogos de la primera mitad del siglo XX se referían, por una parte, al armazón de una disciplina todavía joven y, por otra, a cuestiones puramente formales. Se buscaba la objetividad del relato, la memoria más genuina, el punto de vista del nativo, la representación perfecta de una sociedad: un texto etnográfico debía considerar los cánones de la cientificidad. La forma además tenía que ser cautivadora respetando un orden geométrico de prioridades espacio-temporal. La influencia del editor y el orden dado por el investigador a los hechos permitían purgar los textos de las manchas subjetivas, y el individuo se convertía en el corolario perfecto de sociedades bien compactas, homogéneas, sin brechas étnicas, sociales o religiosas (Calavia Sáez 2006; Franceschi 2006).

A la luz de todo esto, hoy no consideramos las autobiografías indígenas como un género específico (Lejeune 1986) con una forma fija y un esquema cronológico preciso, sino más bien como el resultado de una acción social. Es una "práctica cognitiva» (Clemente 2011: 74) disciplinada de vez en cuando por las diversas demandas que pone en juego. Es un acto que se sitúa más allá del texto. Es una performance que, a la luz de las circunstancias políticas, económi-

2 Para una revisión de estos temas, ver Franceschi (2006).

3 Franz Boas, quien había trabajado en el método y con los textos, y que tanto se esforzó por construir una relación personal y duradera con sus informantes, se pronunció muy claramente sobre el valor epistemológico de biografías y autobiografías. En la revista Science escribía: «They are no facts but memories and memories distorted by the wishes and thoughts of the moment» (Boas 1943: 334335).

4 Pienso, p. ej., en Oakdale (2005), Calavia Sáez (2006 y 2007), Bacigalupo (2010), Bruce y Kopenawa (2010), Course (2011) y Grotti y Brightman (2016). cas y sociales, ahora podemos considerar «como genuinamente indígena» (Calavia Sáez 2006: 184).

Mis reflexiones etnográficas encuentran soporte teórico en algunas experiencias publicadas recientemente por Suzanne Oakdale y Magnus Course y recogidas en el texto Fluent Selves. Autobiography, Person, and History in Lowland South America (2014). Para muchos indígenas contemporáneos la historia se convierte principalmente en una experiencia biográfica (Basso 1995). El relato que se presenta en forma autobiográfica va más allá de la dicotomía historia y mito. También donde el mito sigue presentándose como una suerte de hilo conductor de la sociedad, sus manifestaciones concretas no siguen siendo las mismas.

Trabajé (2005-2017) en Misión Nueva Pompeya en el Chaco argentino, con una comunidad indígena de antiguos cazadores y recolectores. Aunque la mitología es uno de los temas clave de la literatura del área, desde los primeros años de campo me di cuenta que mis informantes no se detenían en este tipo de historias, por otro lado, los relatos de tipo autobiográfico no formaron parte del repertorio clásico de la literatura de área ${ }^{5}$. Quizás esta doble coyuntura me llevó a trabajar con la metodología autobiográfica. La historia que recogí (Franceschi 2018) es la de Teodora Polo, una mujer que tiene hoy unos ochenta años, habla la lengua wichí y un incierto español. Teodora no frecuentó ninguna escuela, no sabe leer ni escribir. Desde el inicio hemos hablado en castellano, que se convirtió en nuestra lengua franca.

Teodora con sus palabras no quiso explicar la génesis del mundo, faltan en su historia correspondencias significativas con los relatos míticos ( $p^{\prime}$ alhalis $)^{6}$, lo que aparece es una pragmática del presente, del pasado y una relación con el futuro sin nostalgia por lo propio perdido, sin resentimiento por los hechos violentos sufridos (Barúa 2008 y 2013). En una trama de incertidumbre, desarraigo y violencia, la urdimbre no presenta ningún matiz moral o victimizador: es una forma de relatar la historia que no quiere crear efectos de suspense, sino consignar una verdad histórica y biográfica; esta historia como las que van surgiendo en el panorama etnográfico de tierras bajas son autobiografías que han colocado a sus prota-

5 Remito a De los Ríos (1980) y Cordeu (2008).

6 El término p'alhalis refiere tanto a las narraciones míticas como a los personajes que toman parte en esas narraciones, que en la wichilogía muchos traducen por 'prototipos'. 
gonistas en un contexto histórico y geográfico preciso permitiendo añadir piezas a una historia oficial aún fragmentaria e incompleta y que los protagonistas quieren que se convierta en historia escrita (Franceschi y Dasso 2010; Montani y Juárez 2015).

\section{LA HISTORIA}

Teodora Polo vive en la comunidad de Misión Nueva Pompeya, en el antiguo cauce del río Bermejo.

Los wichís son un pueblo indígena formado por más de 50000 individuos que viven en la parte semiárida del Chaco central, en la franja formada por los ríos Pilcomayo y Bermejo (provincias de Salta, Chaco y Formosa en la Argentina, y provincia boliviana de Gran Chaco). Se trata de un pueblo que habla su propia lengua, la wichi-Ihämtes (familia lingüística mataco-maka). Sus asentamientos siguen siendo pequeños, homogéneos, relativamente autónomos.

Trabajé con Teodora a partir del año 2005. Hice algunas entrevistas, pero sobre todo pasé mucho tiempo junto con ella, compartiendo lo cotidiano y trabajando juntas. La vi fabricar tejidos, enlazar, en las distintas fases: la elección de la fibra, la preparación del hilo, su tintura, etc. ${ }^{7}$. La observé mientras realizaba distintos tipos de cacharros, eligiendo la tierra, las formas de los contenedores (tinajas, macetas, etc.). Estuve con ella mientras tejía sus mantas de lana con el telar horizontal; en varias ocasiones ella me llevó a visitar a sus familiares cercanos, a través del circuito bien conocido de la visita, siyak. Pasé también mucho tiempo con su esposo (lëch'efwa), hijas (/häse) e hijos (/häs) ${ }^{8}$, con su hermano menor (lëch'inij) y sus hijas (sobrinas, lëwaklani,de Teodora), con los parientes más cercanos del grupo doméstico (lëwet-Iheley), y con una hermana de su padre (tía, lëwit'oj, de Teodora). El resultado fue una historia coral: recompuse la red de relaciones de parentesco de Teodora Polo, la historia de una familia que se hizo sedentaria en los años setenta, los movimientos de bandas y parentelas en la zona sur del Bermejo, chom Iheley.

7 Para un análisis detallado y reciente del trabajo textil, cf. Montani (2017).

8 Los terminos en el texto indican el singular de hijo e hija, el plural es lëles.
Cuando empezó a contarme su historia, un verbo se repetía una y otra vez en descripción de su trabajo: ipätsin (crear, hacer, trabajar). Como sugiere Rodrigo Montani el verbo significa 'crear algo moviendo circularmente un material plástico o flexible', enlazar (que es probablemente la acción prototípica de donde surgió el verbo), hacer tinajas (enrollar en espiral los rollos de arcilla para después coserlos) o levantar una casa con ladrillos (las hileras se van subiendo circularmente, en espiral, y los ladrillos se pegan con un material plástico) ${ }^{9}$.

Teodora tejía, trabajaba y, al hacerlo, recorría la historia de su vida y la de su familia; su tejer (en sentido lato), su trabajo, era la representación material de su existencia, pero ella quería presentarla de nuevo en forma de palabras. Me dio a entender que era una artesana wichí, una de las mejores. Trabajaba con chaguar ${ }^{10}$, arcilla y en el telar. Cuando nuestro vínculo se había convertido en algo más sólido, a pesar de que durante nuestros encuentros seguía trabajando con dedicación y auténtico entusiasmo, comprendí que no estaba interesada en las explicaciones técnicas de los procedimientos. Quería resemantizar la gramática de su trabajo porque era una ocasión para hablarme de manera autobiográfica. Pero había algo más. Durante toda su existencia, cada emoción, inquietud o elaboración de los eventos de su vida había atravesado su cuerpo, se había depositado en sus manos dando forma a todos los productos que vendía o conservaba en casa. Su hacer siempre había anticipado cada una de sus palabras. En todos los objetos que preparaba estaba el sentido más íntimo de lo que le había sucedido. Los artefactos de Teodora se convirtieron en el pre-texto para iniciar una nueva aventura: recordar, contar, explicarse a sí misma lo que le había ocurrido.

Después del nacimiento, en la provincia de Salta, su madre Celia, una mujer wichí abandonada por su marido, deja a Teodora con sus abuelos maternos. Teodora vive sus primeros años con ellos, es una época de sufrimiento, porque el abuelo le mezquinaba todo. N'äyej es el verbo wichí que usa Teodora. En el mundo tradicional wichí la mezquindad tiene connotaciones negativas. El mezquino es simplemente malo y lo que hace es sencillamente feo. La acción realizada por alguien considerado mezquino puede recordar

9 Comunicación personal 3 dic. 2018.

10 Chaguar viene del quechua y es el nombre que dan los criollos a las bromelias chaqueñas en general. 
la actitud del tacaño, tsofwnaj en wichí. El mezquino no comparte, no hace fácil las cosas a los demás, como pueden hacer los dueños del monte con la gente. Tsofwnaj es lo peor que se pueda encontrar en el propio camino. Teodora comentó que mezquinar podía significar también 'controlar, tener cuidado'. A sus ojos parecía una actitud que denotaba premura, cuidado, lo contrario de lo que indica el verbo español. Los abuelos eran mezquinos, estaban celosos de la nieta y no querían que fuera a trabajar con otra gente por miedo a que le sucediera algo. Esto es lo que Teodora quiso traducirme, como si hubiera aprendido con el tiempo a interiorizar y transformar una actitud con connotaciones tan negativas en algo moderado, aceptable. Teodora parecía marcar una complementariedad presente en el mundo wichí entre n'äyej (mezquinar) y $t^{\prime}$ uye (cuidar) ${ }^{11}$ : el dueño del monte mezquina sus animales a los humanos, porque los cuida. Seguramente ella interiorizó la mezquindad de sus abuelos como una acción de protección y tutela.

Teodora se quedó con sus abuelos hasta los diez años, cuando una familia criolla se hizo cargo de ella. Fue una familia que la crió y la amó. Teodora se sentía protegida, pero al mismo tiempo atrapada, ya que este estrecho vínculo, limitaba su libertad, es decir, la libertad de marcharse. Con los criollos aprendió castellano, se capacitó en el trabajo de la lana y de la cría de animales. Los términos que utiliza Teodora para describir su situación son: 'cautiva, huérfana y criada', que traduce al wichí utilizando varias expresiones, todas con distintas connotaciones ${ }^{12}$.

Cuando Teodora tiene unos 17 o 18 años su padre, Eduardo, que hasta aquel momento no había aparecido, la reclama. La niña permanece poco con el padre, porque la maltrató y la violó. Después de una denuncia presentada por la hermana del padre

11 Comunicación personal de Montani 3 dic. 2018.

12 Täskhalhe es utilizado para describir su experiencia como niña y adolescente, cuando pasaba de un grupo a otro. Tänaytsek (pl. tänaytsay) que comenta así: «estos son los prisoneros en la cárcel pero también eramos nosotros cuando ibamos a cosechar el algodon. No teníamos libertad y se tenía que pedir permiso al patrón. Era un lugar cerrado. Un lugar donde uno trabaja no mas. Es una falta de libertad». Esta palabra la utiliza cuando comenta su situación que denomina de cautiverio y para subrayar que fue "criada». Para indicar su condición de huérfana (sin padre) dice, nemek. Esta palabra puede ser asociada a wenek (lëwumek en Montani 2017: 404), utilizada para las personas ancianas botadas, 'abandonadas' y también a kwenek. (lëwit'oj), la madre se hace cargo de ella. Pocos años más tarde Teodora conoce a Bitterman, su futuro esposo. Este es un momento que representa el evento que cambia su destino de joven huerfana/nemek (Franceschi 2012). En más de una ocasión Teodora subrayó que fue ella quien eligió a su marido. Se trata de un dato importante porque el huérfano tradicionalmente no elige a su cónyuge, «no tiene libertad para rechazar a la pareja que le asignan. [...] El huerfano kwenek está sujeto en todo momento a la coerción [...] se le impone el matrimonio con una categoría prescripta de cónyuge» (Palmer 2005: 140-141).

Teodora y Bitterman llegaron a la Misión franciscana entre los años sesenta y setenta, era un periodo económicamente feliz para la Misión porque recién se había fundado una cooperativa de trabajo (Franceschi 2018) y ya los dos tenían allí parte de la familia. Fundaron pronto el actual paraje Araujo, al lado de una laguna, donde viven todavía.

\section{EMOCIONES Y SIGNIFICADOS}

John Leavitt escribió en 1996 un artículo, Meaning and Feeling. Unni Wikan (1992) ya había planteado este tema. Leavitt (1996) empieza con una cita de Hayym Schauss de 1938, un periodista e intelectual que había traducido y comentado partes de la Biblia judía, el Tanaj. Según Leavitt las palabras textuales que comentan el festival judío en el trabajo de Schauss parecen de una precisión que provoca desconcierto, sobre todo cuando se trata de describir las emociones de las mujeres que participan en esta ceremonia. ¿Podría el autor conocer y describir en profundidad cómo y qué sentían estas mujeres? Tampoco Schauss, comenta Leavitt, tenía pretensión teórica. Los antropólogos por lo contrario la tienen, y muy a menudo. El debate sobre la antropología de las emociones gira en torno a comprender si se trata de un ámbito de la naturaleza o de la educación, de ponerlas como factor universal, de nivel biológico, o singular, local, de nivel sociocultural; y, además, si se trata de algo privado, del individuo, o público, de carácter colectivo. Estas son dicotomías que revelan una manera de organizar los conceptos característica del Occidente moderno, y que en última instancia descansa sobre la dicotomía más produnda del determinismo (sociológico, psicobiológico, físico) versus libre albedrío. ¿Dónde poner las emociones? La verdad, según Leavitt, es que las emociones no encajan en ninguna de estas categorías dicotómicas. Las emocio- 
nes involucran el pensamiento y el sentimiento, una sensibilidad, un sentir algo donde el cuerpo juega un papel fundamental porque en cierto sentido nos permite asumir que la lengua o el sistema simbólico son puramente referenciales en sus funciones.

Para definir estados de ánimo como la ansiedad, la alegría o el enojo es necesario asociarlas cada vez y siempre con situaciones culturalmente definidas que van más allá de lo que el estado emotivo provoca. No se trata tanto de comprender cómo una persona siente y piensa sino de intentar ver cómo este sentir y pensar provocan rupturas y escisiones dentro del sistema cultural.

Teodora glosó las palabras pensar, lëtichunlhi, y su pensamiento, tichunhayaj, con 'algo que sale de tu mente, pensando siempre, sin parar'. En su universo se trata de una acción que le provoca fatiga, cansancio físico y un continuo darse vuelta. Un estado que no le permite vivir en paz y tranquilidad. Pensar en wichí es un verbo que introduce una acción que requiere un tiempo para reflexionar ${ }^{13}$. La condición más apremiante en la que se encontraba era el hecho de estar continuamente preocupada y pensativa, un estado que ella describía utilizando la palabra tichinlhi, un término, me dijo, que había que situar entre la reflexión y el pensamiento, lëtichunhayaj, un estado de ánimo que no permite vivir con tranquilidad, lëtamsek. El pensamiento expresado en el sustantivo lëtichunhayaj refleja una actitud que puede tener connotaciones tanto positivas como negativas. Se trata de un pensamiento constante y continuo que a menudo puede ser asociado a la nostalgia, a 'echar de menos' algo o a alguien.

\section{EtNOGRAFÍA}

Construir la historia genealógica y las dinámicas de parentesco me permitió comprender cómo Teodora trabajó con las emociones y sus significados. Teodora recordó a menudo eventos que crearon quiebres. Lo

13 Tichun es una raíz polisémica, 'pensar, recordar, añorar'. Tichunlhi está con el durativo, -lhi, y entonces es como 'estar pensando'. Lëtichunhayaj (sustantivo con prefijo) es 'su pensamiento' y se usa, entre otras cosas, para decir que alguien tiene nostalgia, añoranza (por el ser amado, p. ej.) o tristeza (por el deudo, incluso es la palabra que traduce 'duelo'). El significado de tichunche, 'pensar en algo' se determina según la diversidad de contextos. Comunicación personal de Montani 3 dic. 2018. hizo con gran naturalidad. Nunca se detuvo en complicadas explicaciones sobre los lazos familiares o las elecciones matrimoniales, para ella, en el universo wichí, estas últimas son dadas, y poca veces negociables. El matrimonio de su hermano Roque con la mujer de uno de sus hijos; el abandono inesperado de la nieta Yolanda; los divorcios repentinos y los continuos adulterios eran narrados sin mayores comentarios, desapasionadamente. Lo que Teodora quizás intentaba hacer era más complejo: no tanto acercarse con una reflexión emotiva a las costumbres culturales, sino llenar las normas culturales de las razones emotivas que en su opinión provocaban un conflicto que acarreaba dolor. El conflicto pertenecía al grupo, a menudo se trataba de alianzas con afines que ella consideraba demasiado cercanos, y se resolvía o perduraba, en su interior. No era su resolución lo que le interesaba a Teodora, a ella le urgía ordenar emotivamente una aflicción que era íntima y subjetiva, y para la cual la cultura daba disposiciones relativas, que permanecían en el ámbito de gestión del grupo doméstico.

La norma de residencia postnupcial que siguen los wichís es uxorilocal (la nueva pareja se va a vivir con los padres de la mujer) y la descendencia es bilateral (tanto materna como paterna). Los wichís no tienen una regla prescriptiva para formar las alianzas, pero sí una estrategia preferencial que les permite mantener un delicado equilibrio entre exogamia y endogamia. El ideal es que el sujeto contraiga matrimonio con un miembro de una parentela con el cual su propia parentela ya ha establecido alianzas matrimoniales. Dicho en términos simples, el ideal es casarse con un alguien que es ya un pariente político. Esto es lo que se conoce como principio de afinidad serial, mediante el que se crean vínculos de afinidad en una forma concatenada y en diferentes generaciones ${ }^{14}$.

En este contexto fue que el hermano de Teodora, Roque (hijo de su madre con un segundo esposo), se había casado con Dolores, la esposa (desde entonces, exesposa) de Donato, uno de los hijos de Teodora. La esposa potencial de Roque se había convertido en la madre de su sobrina Yolanda, con la cual la familia Gómez ya tenía un vínculo precedente porque el hermano de Donato, Alberto, ya se había casado con Bonifacia, una de las hermanas de Dolores. Esta mu-

14 Sobre el tema del parentesco, cf. Braunstein (1983), Palmer (2005), Barúa (2007), Bossert, Sendón y Villar (2009) y Montani (2017). 
jer, antes de que Roque se convirtiera en su marido, lo consideraba lëchäti (el término puede traducirse como 'abuelo', 'suegro' o 'tío paterno') y Roque consideraba a Dolores letiche (el término traduce 'cuñada' o 'nuera'); Dolores entraba por tanto en la categoría de parientes denominados lëfwayenek, un término que se usa para los parientes afines: el yerno, el cuñado; lëfwayenek define la categoría de receptores de esposa mientras que lëtiche la de los receptores de esposo ${ }^{15}$.

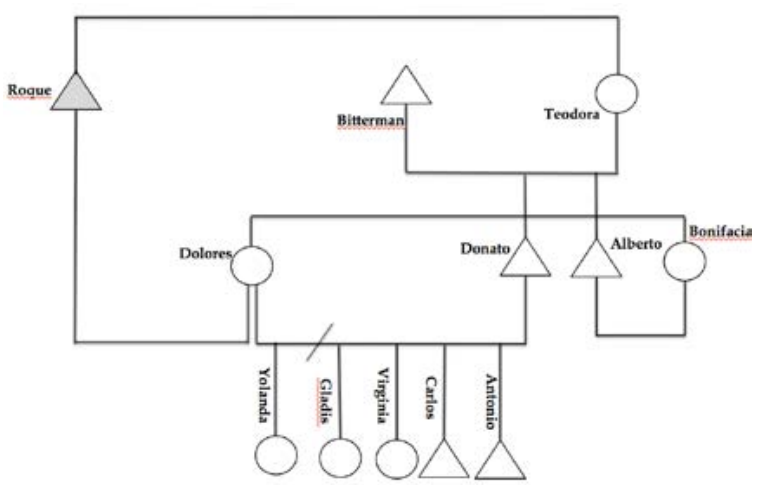

¿De qué manera encajar y luego decantar las consecuencias emocionales de todas las conductas que podrían causar vergüenza, dolor, sensación de incomodidad, ira y frustración? ¿Cómo vivir y administrar un universo cultural de relaciones de parentesco compuesto por reglas aparentemente tan sólidas? Nunca me había detenido a reflexionar sobre esto, pero sí era cierto que el universo del parentesco wichí se construía según reglas que no se podían discutir ni repensar y esto representaba la forma más habitual de hacer cultura.

Teodora me había mostrado y dejado en claro gracias y durante su relato autobiográfico, que aquellas normas culturales según las cuales se formaban alianzas con afines cercanos, durante diferentes generaciones y entre diferentes clases de edad, producían reacciones inevitables, que también podían causar daño y dolor especialmente cuando se acercaban al peligro de la endogamia. Era un repertorio emocional que Teodora no había podido ordenar ni canalizar. Teodora creó su archivo emocional ${ }^{16}$. Creo que su intento fue comprender hasta qué punto llegaba la

15 Los términos afines en wichí se diferencian entre los donantes y los receptores de cónyuges (Palmer 2005: 135).

16 Sin detenerme en la etnografía feminista quiero señalar las reflexiones de Martha Nussbaum y Nancy ScheperHughes que buscan en sus trabajos un punto de encuentro fuerza de la cultura, hasta dónde era posible marcar sus límites y fronteras, las zonas de luz y las de sombra. Teodora veía su cultura desde arriba, por así decirlo. Incluso hubo elecciones que causaron rupturas temporales de los vínculos entre los distintos grupos familiares, y el hecho de que ella regresara sobre el mismo tema, comentándolo, significaba que no solo no podría haber evitado esas fracturas, sino que, en cualquier caso, era algo que ya no hubiera podido recomponerse.

Alianzas preferenciales, cíclicamente repetidas, con un buen margen de libertad (nunca se trata de alianzas prescriptas, sino alianzas dentro del rango de lo que no está condenado o excluido) que provocan una ruptura inesperada, pero habitual. Era un modelo sólido pero flexible, que se consolidaba mediante la repetición de ciertas reglas y que continuaba, inexorable e independientemente. Teodora quería ayudarme a comprender no solo el funcionamiento del sistema de alianzas matrimoniales, sino también a mirar dentro de sus engranajes.

A menudo, todo esto fue descrito por ella como doloroso, o más bien era una cuestión de constante preocupación, lëtichunlhi. De hecho, parecía que el peso recaía sobre ella. Ella tejió alianzas, aflojó, reparó o fortaleció donde lo consideró necesario. Es exactamente en este sentido que Teodora profundizó conmigo sus pensamientos y emociones. La suya fue una relectura desde arriba, y una mirada centrada en las emociones que me permitió llegar a comprender un poco más tanto a través del relato auto-biográfico la sociedad wichí.

\section{BIBLIOGRAFÍA CITADA}

Bacigalupo, Ana. 2010. «The Life, Death, and Rebirth of a Mapuche Shaman: Remembering, Forgetting and the Willful Transformation of Memory». Journal of Anthropological Research 66(1): 97-119.

Barúa, Guadalupe. 2007. Un arte delicado. Relaciones entre el parentesco, el conflicto y el acontecimiento entre los wichí del Chaco Central. Buenos Aires: Editorial Dunken.

Barúa, Guadalupe. 2008. "Las sociedades de nostalgia como un tipo de sociedad: el caso Wichí», en VI Congreso Argentino de Americanistas, tomo 3: 15-33. Buenos Aires: Sociedad Argentina de Americanistas.

entre la vida y sus representaciones sin caer en ningún esencialismo feminista. 
Barúa, Guadalupe. 2013. «La expresión de la Nostalgia wichí a traves de los cantos lamentos de las mujeres», en Tola Florencia, Medrano Celeste y Cardin Lorena (eds.), Gran Chaco. Ontologías, Poder, Afectividad: 213-231. Buenos Aires: Ethnographica.

Basso, Ellen. 1995. The Last Cannibals. A South American Oral History. Austin: University Texas Press.

Boas, Franz. 1943. «Recent Anthropology». Science 8: 311-337.

Bossert Federico, Pablo Sendón y Diego Villar. 2009. «Relevancia de las teorías clasicas del parentesco para el estudio de las sociedades amerindias (Andes Centrales, Amazonía y Gran Chaco)", en VI Congreso argentino de Americanistas, tomo 3: 35-64. Buenos Aires: Sociedad Argentina de Americanistas.

Braunstein, José. 1983. "Algunos rasgos de la organización social de los indígenas del Gran Chaco». Trabajos de Etnología 2: 9-102.

Bruce, Albert y Davi Kopenawa. 2010. La chute du ciel. Parole d'un chaman yanomami. París: Terre Humaine.

Brumble, David. 1985. «Sam Blowsnake's Confessions: Crashing Thunder and the History of American Indian Autobiography». Canadian Review of American Studies 16: 271-282.

Brumble, David. 1988. American Indian Autobiography. Berkeley: University of California Press.

Calavia Sáez, Oscar. 2006. "Autobiografia e sujeto histórico indígena». Novos Estudios 76: 179-195.

Calavia Sáez, Oscar. 2007. «Autobiografía e liderança indígena no Brasil». Tellus 12: 11-32.

Clemente, Pietro. 2011. "L'antropologia che intervista. Le storie della vita», en Massimo Pistacchi (ed.), Vive Voci. L'intervista come fonte di documentazione: 73-89. Roma: Donzelli.

Cordeu, Edgardo. 2008. El origen de la pintura. Mitología, memoria étnica y autobiografía del artista indígena Ógwa, vol. 6. Asunción: Centro de Estudios Antropológicos de la Universidad Católica.

Course, Magnus. 2011. Becoming Mapuche. Person and Ritual in Indigenous. Urbana: University of Illinois Press.

De los Ríos, Miguel Angel. 1980. «Una historia de vida: notas para la hermenéutica del ciclo vital de la etnia mataco». RUNA 13(1-2): 79-112.
Franceschi, Zelda Alice 2006. Storie di vita. Percorsi nella storia dell'antropologia americana. Bolonia: Clueb.

Franceschi, Zelda Alice. 2012. "Le buone mani e le mani buone di Teodora Polo: una storia di vita», en Franceschi, Zelda Alice (coord.), Storie di vita: 43-81. Monográfico de Annuario di Antropologia 14. Milán: Ledizioni.

Franceschi, Zelda Alice. 2018. Tessere storie. Etnografia nel Chaco argentino. Bolonia: Emil.

Franceschi, Zelda Alice y María Cristina Dasso. 2010. Etnografías. La escritura como testimonio entre los wichi. Buenos Aires: El Corregidor.

Grotti, Vanessa y Marc Brightman. 2016. «Narratives of Invisibles: Autobiography, Kinship, and Alterity in Native Amazonia». Social Analysis 60(1): 92-109.

Krupat, Arnold. 1985. For Those Who Come After. A Study of Native American Autobiography. Berkeley: University of California Press.

Leavitt, John. 1996. "Meaning and Feeling in the Anthropology of Emotions». American Ethnologist 23: 514-523.

Lejeune, Philippe. 1986. I/ patto autobiografico. Bolonia: II Mulino.

Montani, Rodrigo. 2017. El mundo de las cosas entre los wichís del Gran Chaco. Un estudio etnoligüístico. Cochabamba: Instituto de Misionología.

Montani, Rodrigo y Gerardo Juárez. 2015. Los días del pasado. Historias de los wichí de Morillo, San patricio, Los Baldes. Mahnayay ta iyejen p'ante wichi ta yhi Muliyus San Patlisyu Ihoya Kale-hí. Córdoba: Ediciones La Marmosa.

Oakdale, Suzanne. 2005. I Foresee My Life: The Ritual Performance of Autobiography in an Amazonian Community. Lincoln: University of Nebraska Press.

Oakdale, Suzanne y Magnus Course (eds.). 2014. Fluent Selves. Autobiography, Person and History in Lowland South America. Lincoln: University of Nebraska Press.

Palmer, John Hilary. 2005. La buena voluntad wichí: una espiritualidad indígena. Buenos Aires: Grupo de Trabajo Ruta 81.

Wikan, Unni. 1992. "Beyond the Words. The Power of Resonance». American Ethnologist 19: 460-482. 